\title{
MALIGNANT HYPERPYREXIA: A THERAPEUTIC AND INVESTIGATIVE REGIMEN
}

\author{
J.E.S. Relton, F.F.A.R.C.s. (ENG.), 'D D.J. Stewahd, F.R.C.P.(c), \\ R.E. CReighton, F.R.C.P.(c), ${ }^{\circ}$ AND B.A. BRitT, F.R.C.P.(c) $\dagger$
}

LE sUCCÈs DU TRATTEMENT CLINQue du syndrome d'hyperthermie maligne dépend, en premier lieu, de la précocité du diagnostic. Il est essentiel d'instituer un traitement intensif avant que la $\mathrm{T}^{\circ}$ de l'organisme soit trop élevée ou bien établie. Un diagnostic tardif et un traitement inadéquat sont des facteurs importants dans linsuccès du traitement.

Un des auteurs (B.A.B.) a recueilli les détails diau-delà de plus 300 cas d'hyperthermie maligne; le taux de mortalité dépassait 60 pour cent. Une analyse détaillée de 89 de ces cas ( 1 ), a démontré une correlation fortement positive entre le niveau maximum de température atteint et la mortalité. Six cas dont la température maxima est demeurée inférieure à $102.9^{\circ} \mathrm{F}$, ont recouvré la santé et, chez ces cas, lanesthésie a été discontinuée en-decà de quelques minutes après l'induction.

Le pourcentage de survivance diminue à mesure que la température atteinte augmente (voir tableau I).

TABLEAU I

NOMBre D'ANESThÉsies

\begin{tabular}{crrrr}
\hline \hline $\begin{array}{c}\text { Température maxima } \\
{ }_{\mathbf{F}}\end{array}$ & More & Survivant & Total & $\begin{array}{r}\text { Pour cent de } \\
\text { survivant }\end{array}$ \\
\hline $99.0-102.9^{\circ} \mathrm{F}$ & & 6 & 6 & 100 \\
$103.0-106.9^{\circ} \mathbf{F}$ & 9 & 14 & 23 & 61 \\
$107.0-110.9^{\circ} \mathbf{F}$ & 37 & 8 & 45 & 18 \\
$110^{\circ} \mathbf{F}$ & 5 & 1 & 6 & 17 \\
Non specifié & 6 & 8 & 14 & 57 \\
Totaux & 57 & 37 & 94 & 36 \\
\hline
\end{tabular}

De façon à faciliter le diagnostic précoce et le traitement du syndrome de l'hyperthermie maligne, nous suivons, à l'hôpital "Sick Children" Toronto, le protocole suivant. Il résume les premiers signes clíniques du syndrôme il décrit les moyens de vérification et de traitement et il renseigne sur l'endroit où sont placés l'équipement spécial et les médicaments susceptibles d'être employés. Nous avons placé des copies de ce protocole dans toutes les salles d'opération pour y recourir si nécessaire.

\section{Photocole en cas d'Hypertherme}

A Identification du syndrome

Habituellement, il apparaît:

(1) Une hypertonie des muscles squelettiques qui survient

- Department of Anaesthesia, University of Toronto and Hospital for Sick Children, Toronto. tDepartment of Anaesthesia and Department of Pharmacology, University of Toronto. 
(a) Soit immédiatement après l'adininistration de la succinylcholine. Si, après l'administration de succinylcholine, il ne survient pas de relâchement musculaire, il s'impose de retarder la chirurgie et de faire une nouvelle évaluation du malade.

(b) Soit plus tard au cours de l'anesthésie après l'usage d'agents puissants comme lhalothane.

(2) Une tachypnée, une tachycardie, une transpiration, une cyanose ou une chaleur plus élevée qu'à lordinaire de la chaux et, cela, sans explication, tout cela, sont des signes précoces mais non spécifiques.

(3) Une élévation de la température du malade $\left(1^{\circ} \mathrm{C}\right)$.

Si l'on considère le pronostic, il est fortement désirable que le syndrome soit reconnu avant que la réaction pyrexique soit forte et bien établie. Une élévation de la température de lorganisme peut être un signe tardif.

\section{B Mesures thérapeutiques}

(1) Immédiatement, arrêter la chirurgie et ladministration de tout agent puissant par inhalation et de tout myorésolutif.

(2) Hyperventiler le malade avec un mélange riche en oxygène ou de l'oxygène à 100 pour cent si nécessaire. Se servir d'une machine ne contenant aucune trace de vapeur anesthésique.

(3) Le traitement médicamenteux doit commencer immédiatement.

(a) Des bicarbonate de sodium (7.5\%) par voie endoveineuse $4 \mathrm{ml} / \mathrm{kg}$ immédiatement et répéter selon les résultats du sang artériel.

(b) Des chlorhydrate de procaïne ( $1 \%$ ) IV chez les malades présentant une hypertonie des muscles squelettiques. La dose d'attaque est $3-4 \mathrm{mg} / \mathrm{kg}$ suivie d'un goutte-à-goutte de $0.2 \mathrm{mg} / \mathrm{kg} / \mathrm{min}$. L'infusion de procaïne doit être surveillée par lélectroencéphalogramme (2).

(c) Du mannitol - 1 gramme/kilo v.

(4) Commencer le refroidissement actif. Placer le malade sur une toile caoutchoutée, appliquer des sacs de glace, de l'eau glacée et des éventails. On peut également envisager l'utilité du refroidissement intragastrique et de lavements froids.

(5) Installer une canule dans une veine et administrer une solution de lactate Ringer refroidie.

\section{Moyens de recherche}

(1) Surveiller au moyen de:

un stéthoscope oesophagien

un manchon à pression artérielle

un électracardiogramme

un thermomètre à canaux multiples (rectal, oesophagien et musculaire)

(2) Installer une canule artérielle pour prélèvements.

(3) Prélever immédiatement $10 \mathrm{ml}$ de sang et demander:

les gaz artériels

les électrolytes ( $\mathrm{na}, \mathrm{K}, \mathrm{Cl}, \mathrm{Ca}_{3} \mathrm{PO}_{4}$ ) 
creatine phosphokiniase (C.P.K.)

sGOT et LDH

Corriger tout déséquilibre électrolytique selon les résultats des analyses. On peut s'attendre à trouver une hypocaliémie et une hyperkaliémie suivie d'une hypokaliémie.

(4) Faire des analyses de sang artériel \& toutes les 10 minutes et faire les corrections selon les résultats.

(5) Mettre une sonde et, dans l'urine, rechercher l'hémoglobine et la myoglobine. Mesurer le débit urinaire.

D Ou se trouve le nécessaire pour de tels cas

(Donner ici, dans le protocole, tous les renseignements sur l'endroit où sont placés, dans le bloc opératoire, l'instrumentation et les médicaments spéciaux suivants).

Une machine ne contenant aucune trace de vapeur d'agent anesthésique, c'est-àdire, sans vaporisateur.

Un thermomètre à canaux multiples.

Une toile caoutchoutée, des sacs de glace, de la glace et des éventails électriques.

Un appareil pour refroidissement intragastrique.

Médicaments: Mannitol, procaïne, lactate Ringer refroidi et du bicarbonate de sodium et du gluconate de calcium.

\section{Résumé}

Si l'on fait le diagnostic précoce du syndrome de lhyperthemie maligne et que l'on institue un traitement vigoureux et agressif, on pourra s'attendre a une dimimution du taux de mortalité. Il est suggéré de rédiger un résumé des signes précoces du syndrome et une description des moyens de recherche et de traitement employés pour en distribuer une copie aux anesthésistes dans toutes les salles d'opération. 